\title{
The youth mental health crisis during the COVID-19 pandemic: The role of school closures
}

Christina Felfe ( $\nabla$ christina.felfe@uni-wuerzburg.de)

University of Würzburg https://orcid.org/0000-0002-4095-8112

Judith Saurer

University of Wuerzburg

Patrick Schneider

University of Wuerzburg

Judith Vornberger

University of Wuerzburg

Valentin Klotzbuecher

University of Freiburg

Michael Erhart

ASH Berlin

Anne Kaman

UKE Hamburg

Ulrike Ravens Sieberer

University Clinic Eppendorf

\section{Social Sciences - Article}

Keywords:

Posted Date: March 7th, 2022

DOI: https://doi.org/10.21203/rs.3.rs-1304103/v2

License: (c) (i) This work is licensed under a Creative Commons Attribution 4.0 International License.

Read Full License 
The youth mental health crisis during the COVID-19 pandemic: The role of school closures

Christina Felfe ${ }^{1,2,3}$, Judith Saurer ${ }^{1}$, Patrick Schneider ${ }^{1}$, Judith Vornberger ${ }^{1}$, Valentin Klotzbücher ${ }^{4}$, Michael, Erhart $^{5}$, Anne Kaman ${ }^{5}$, Ulrike Ravens-Sieberer ${ }^{5}$

\begin{abstract}
:
The youth mental health crisis has become so acute during the COVID-19 pandemic that a coalition of U.S. pediatric health experts has declared it a national emergency ${ }^{1}$. To which extent this crisis can be attributed to the pandemic or the different pandemic measures is unknown. School closures are among the most heavily debated pandemic measures, with insufficient evidence on their costs ${ }^{2,3,4,5}$. We seek to narrow this blind spot, by compiling data on the manifold school closure and re-opening strategies implemented across the federal states of Germany at the onset of the pandemic and combining it with data from a nationwide, population-based, longitudinal survey on youth mental health and the largest crisis helpline for children and adolescents. We show that prolonged school closures led to a significant deterioration in youth health-related quality of life, precipitating first signs of mental health problems. Effects were most severe among boys, less mature adolescents, and families with limited living space. Young people also increasingly sought support from crisis helplines to discuss arising family and friendship problems. Importantly, the effects persist even when abstracting from the overall strain imposed by the pandemic or further pandemic measures. Indeed, school closures explain around two thirds of the aggravation of the youth mental health crisis throughout the first pandemic wave and there is no sign for a swift recovery.
\end{abstract}

The rapid spread of the novel coronavirus SARS-CoV-2 and the associated respiratory disease COVID-19 motivated governments around the globe to impose drastic policy measures such as physical distancing, contact reduction, working from home or homeschooling. The pandemic and its related measures massively affected people's life and left scars on people's mental health ${ }^{6,7,8}$. While the virus disproportionally affected the elderly, negative psychological consequences were particularly pronounced during childhood and adolescence, the most dynamic and thus vulnerable period in human life from the perspective of developmental psychology $y^{9,10,11}$. Globally, child and adolescent mental health problems are at unprecedentedly high levels. Recent studies report a doubling of child and adolescent anxiety and depression levels, compared with pre-pandemic estimates ${ }^{12}$. Worldwide, at least $13 \%$ of people between the ages of 10 and 19 now live with a diagnosed mental health disorder ${ }^{13}$. Despite these alarming numbers, the role played by the different aspects of the pandemic, in particular its related measures, are unknown. We seek to narrow this blind spot by directing the attention to the role played by school closures.

School closures were among the first measures taken to fight the viral spread. By mid-March 2020, when the WHO classified the COVID-19 as a pandemic, governments of 109 countries had mandated partial or full school closures ${ }^{14}$. By mid-April 2020, more than 1.13 billion children and adolescents around the globe, corresponding to $72 \%$ of all enrolled learners, were exempted from in-person education ${ }^{14}$. Schools are children's and adolescents' place to learn, but also their place to engage and establish social interactions. For children and adolescents in need, schools represent the first point of contact and guard their wellbeing ${ }^{4}$. Thus, school closures implied a dramatic disruption to children's and adolescents' lives, during a phase of life when engaging, predictable environments and stable, positive social interactions are crucial for promoting children's and adolescents' socio-emotional development and preventing challenging behaviors ${ }^{15}$.

\title{
Results
}

\section{Ad-hoc decisions about school closures}

Disentangling the overall impact and singling out the consequence of school closures, and thus of one specific pandemic measure is challenging. We zoom in on one country, the Federal Republic of Germany, where the federal states enjoy cultural and educational sovereignty. Thus, we observe a large array of school closure and re-opening strategies, orthogonal to the mental health consequences caused by the overall course of the pandemic and other pandemic measures, and thus providing us with an ideal identifying source of variation.

In international comparisons, Germany registered rapidly increasing COVID-19 case rates at the outbreak of the pandemic ${ }^{16}$. In reaction to the exponentially growing case rates, all 16 German federal governments mandated state-wide school closures on March 16, 17, or 18, 2020. From April 20, 2020, onwards, the states reopened schools gradually, but each state followed its own strategy guided by various arguments from education

\footnotetext{
${ }^{1}$ University of Würzburg, Würzburg/ Germany

${ }^{2}$ Center of Economic Performance, London/ United Kingdom

${ }^{3}$ CESifo, Munich/Germany

${ }^{4}$ University of Freiburg, Freiburg im Breisgau/ Germany

${ }^{5}$ University Medical Center Hamburg-Eppendorf, Hamburg/Germany
} 
science and developmental psychology. Generally, priority was given to graduating cohorts (depending on the state, grade level 4 or 6 for primary school, and depending on the school track, grade level 9, 10, 12 or 13 for secondary school). The return of the remaining grade levels was organized stepwise and varied across states. Some states followed a strict chronological order drawing in first the graduating cohorts followed then successively by the younger ones, while others gave priority to entry-level grades followed by the intermediate ones. Thus, the number of weeks an individual was exempted from in-person learning depended on her state of residence, the grade level she belonged to, and the school track she attended ${ }^{17}$.

\section{Systematic processing of school re-opening strategies}

In the absence of a comparative and transparent overview, we processed all state-specific Corona protection ordinances and compiled a dataset on the state-specific school closure and re-opening strategies ${ }^{17}$. Figure 1 , Panel a illustrates the closure and re-opening strategies adopted by the 16 German federal states, differing in the timing, the intervals, and the overall duration of the re-opening phase. Figure 1, Panel b displays the resulting variation in school closures by grade levels (subsuming the variation across school tracks, see Extended Data Figure 1 for the variation by school track). Two facts stand out: i) the priority given to the graduating students who started to return already after 4.7 weeks; ii) the substantial variation within grade levels (ranging from 4.7 to 13 weeks).
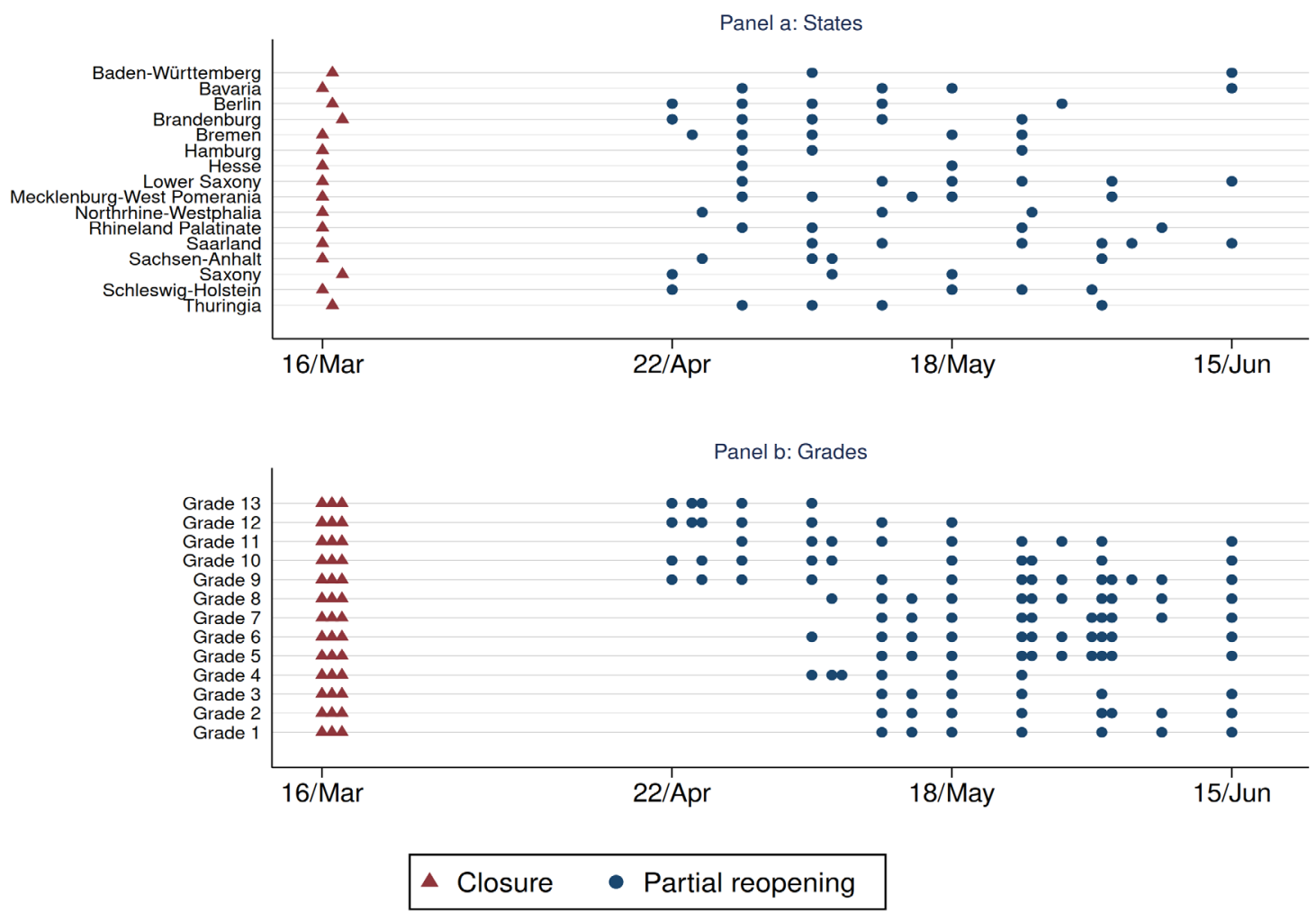

Figure 1: Variation in school closure and re-opening strategies by states (Panel a) and school grades (Panel b). Data are compiled by screening the state-specific Corona protection ordinances. Each blue dot represents the date when the respective federal government (partially) re-opened schools for selected grade levels, possibly restricted to the grade levels of certain school tracks (Panel a) and the resulting variation in the re-opening dates by grade-levels across the federal states and possibly school tracks (Panel b). As the Extended Data Figure 1 shows, substantial variation remains when distinguishing between the different school tracks.

\section{Identifying the mental health impact of school closures}

The described variation in the duration of school closures within states and within grade levels (differentiated by the school track) provides us with an ideal source of variation to identify the mental health consequences of prolonged school closures. We will leverage this variation and compare the mental health of adolescents residing in the same state but attending different grade levels (possibly also different school tracks). Doing so allows us to absorb the state-specific level of adolescents' mental health subsuming any deterioration in adolescents' mental health because of the severity of the pandemic (e.g., causing fear or insecurity) or the stringency of the pandemic measures (e.g., various degrees in contact reductions, closure of leisure or sports facilities). At the same time, we control for grade level or school track-specific differences in mental health that may arise because 
adolescents attending different grade levels or school tracks may struggle or cope differently with the pandemic and its related measures. This empirical approach is known as difference-in-difference strategy, or more generally as two-way-fixed effects approach and described in more detail in the method section ${ }^{18,19}$.

The following example helps illustrating the empirical approach. Bavaria gave priority to entry grade levels with the higher grade levels following only subsequently. Thus, in Bavaria a $5^{\text {th }}$ grader (the entry grade level in secondary school) returned to school by May 18, 2020, while a $6^{\text {th }}$ grader returned only by June 15, 2020 (4 weeks later). In contrast, the neighboring state Baden Württemberg re-opened schools for all lower grade levels in secondary schools $\left(5^{\text {th }}, 6^{\text {th }}, 7^{\text {th }}\right.$, and $8^{\text {th }}$ graders) "en bloc" on June 15,2020 . The first difference between $5^{\text {th }}$ and $6^{\text {th }}$ graders in Bavaria allows holding state-specific effects of the pandemic and its related measures constant, leaving us with the mental health differences because of the additional 4 weeks school closures, but likely also because of age-differences in mental health or in the way how differently aged adolescents dealt with the pandemic and its measures. The second difference between $5^{\text {th }}$ and $6^{\text {th }}$ graders in Baden Württemberg allows determining the age-differences in mental health during the COVID-19 pandemic, again holding state-specific effects of the pandemic or its related measures constant. The difference-in-differences then isolates the effect of 4 additional weeks school closure net of state- and age-specific differences in adolescents' mental health during the COVID-19 pandemic. We will turn to potential mechanisms underlying the effect of prolonged school closures during the COVID-19 pandemic at the end of the article.

\section{Nationwide data on youth mental health}

Germany offers unique, nationwide, population-based data on adolescents' mental health during the COVID-19 pandemic (COvid-19 and PSYchological health, short COPSY). The COPSY data represents the perspective of the adolescents and was collected via an online survey among $n=1$ '040 11-17-year-olds between May 26 and June 10,2020 . Follow-up surveys with $n=1^{\prime} 077$ adolescents took place between December 17, 2020, and January 25, 2021 (Response rate: $85.1 \%$ plus sample increase), and with $n=1^{\prime} 139$ adolescents between September 14 , and October 11, 2021 (Response rate: 73.7\%, plus sample increase). In addition, $n=1^{\prime} 586\left(n=1^{\prime} 625, n=1^{\prime} 618\right)$ parents of 7- to 17-year-olds participated in the (follow-up) surveys. Sampling weights allow us to respect the population structure and scale our results to make representative and policy-relevant statements ${ }^{20,21}$.

The survey included internationally established and validated instruments for measuring adolescents' healthrelated quality of life (HRQoL) using the KIDSCREEN-10 Index ${ }^{22}$ and screening instruments for mental health problems, such as the HBSC Symptom Checklist (HBSC-SCL) to check for psychosomatic complaints ${ }^{23}$, the Strengths and Difficulties Questionnaire (SDQ) to elicit behavioral and emotional difficulties ${ }^{24}$, the Center for Epidemiological Studies Depression Scale (CES-DC) ${ }^{25}$ and the Screen for Child Anxiety Related Disorders (SCARED) ${ }^{26}$ scale to measure levels of depressive and anxiety symptoms, respectively. Crucially, COPSY builds upon and can be referenced to the BELLA cohort study ( $n=1^{\prime} 556$ in 2017), the mental health module of the nationwide, longitudinal, representative German National Health Interview and Examination Survey for Children and Adolescents (KiGGS) $20,21,27,28,29$. Thus, we can determine the overall deterioration in adolescents' mental health over the course of the COVID-19 pandemic and quantify the contribution of the school closures. Merging COPSY Wave 1 with the data on school closures (by state of residence, grade level, and school track) results in a sample of $n=907$ 11-17-yearold adolescents, attending grade levels 4 to 13 and having experienced between 4.7 and 10.1 weeks of school closure up to the time of the survey. It allows for a snapshot of youth mental health towards the end of the first lockdown and for an analysis of the short-run mental health effects of prolonged school closures (3.95 weeks on average taking into account that each adolescent was exempted from school for at least 4.7 weeks). Data from the follow-up surveys provide us with a glimpse on the development of youth mental health at different points in time over the course of the pandemic.

\section{School closures cause mental health issues in the short run}

Figure 2a summarizes the estimated short-run effect of school closures on adolescents' HRQoL and symptoms of mental health problems. One additional week of school closures decreases adolescents' HRQoL by 0.112 standard deviations (henceforth $\mathrm{sd})(P<0.001)$ and increases psychosomatic symptoms by $0.071 \mathrm{sd}(P<0.05)$, behavioral and emotional health problems by $0.083 \mathrm{sd}(P<0.05)$, and depressive symptoms by $0.073 \mathrm{sd}(\mathrm{P}<0.05)$. No effect is found for anxiety symptoms. Extended Data Table 2 shows the baseline results as well as the results of a battery of sensitivity checks. Most importantly, we i) tighten our specification and use within school track variation in the duration of school closures only, ii) allow for a non-linear impact of the duration of school closures, iii) rely on an alternative imputation method for the duration of school closures in case of missing survey dates, iv) adjust the duration of school closures for school holidays, v) use adolescents' self-reports on home schooling, and vi) draw upon parental reports of adolescents' mental health. 


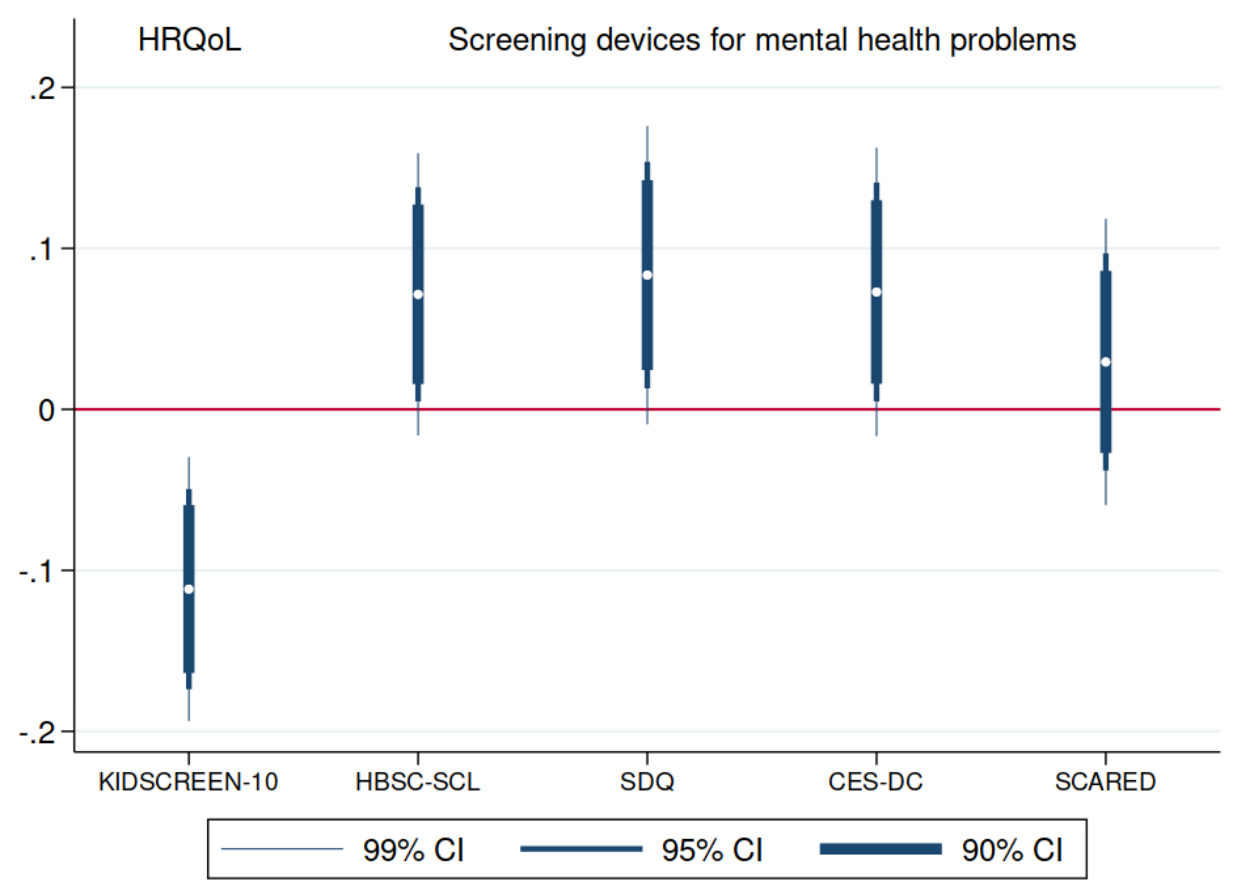

b
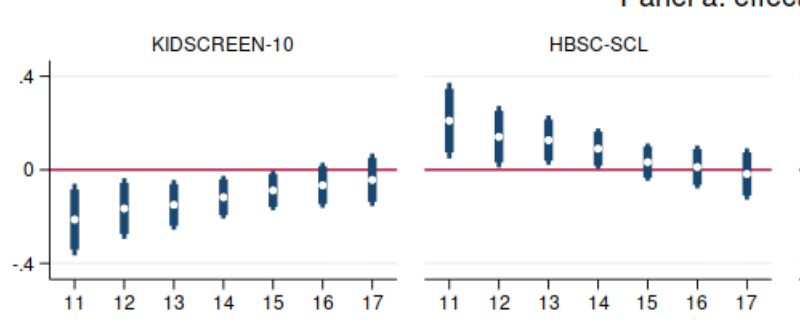

Panel a: effect by age

Panel b: effect by gender
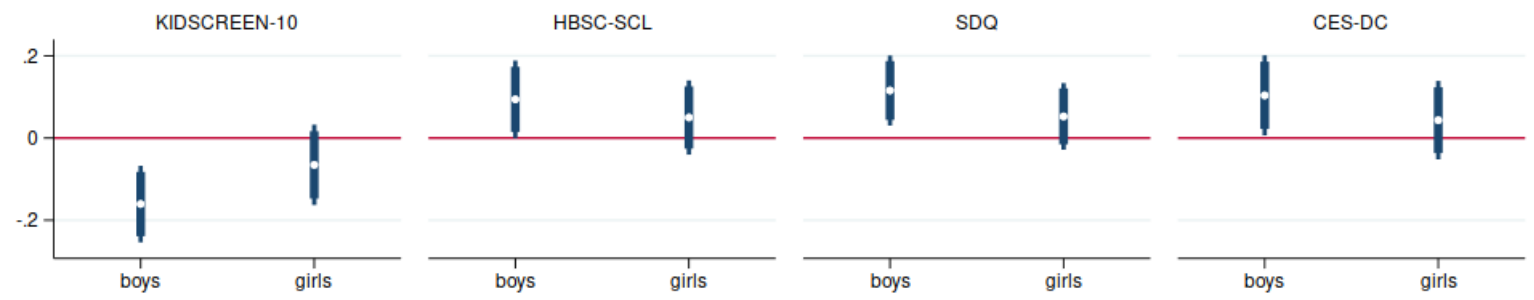

Panel c: effect by living space per child
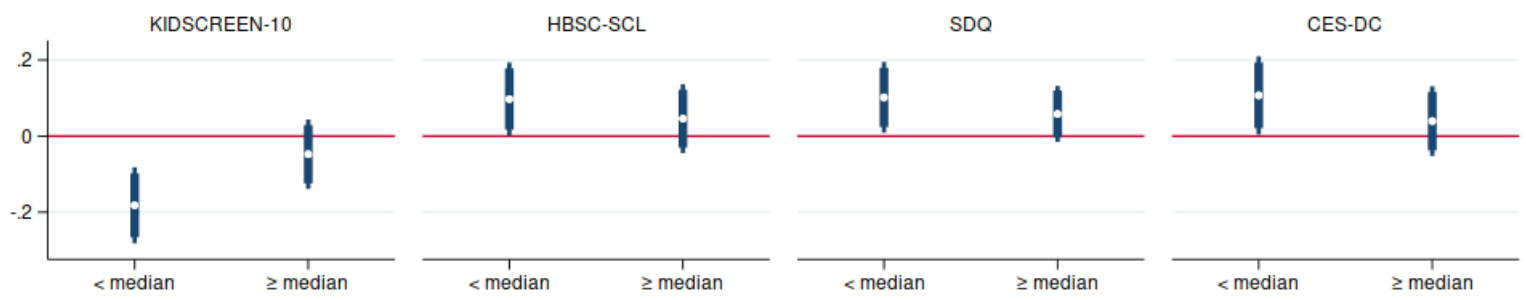

Figure 2a b: Short-run impact of school closure on well-being and mental health Estimates (including the $99 \%, 95 \%$ and $90 \%$ confidence interval) for the effect of one additional week of school closure on standardized measures (mean 0, std. 1) of adolescents' self-reported HRQoL (Panel a: KIDSCREEN-1), and screening instruments for mental health problems (Panel b: HBSC-SCL, SDQ, CES-DC and SCARED). For all scales, except KIDSCREEN-10, higher values express more health problems. Each bar results from a separate two-way fixed effect regression (controlling for a set of state and school track-specific grade level fixed effects as well as for age and gender) using ordinary least squares with sampling weights and standard errors clustered at the state*grade level*school track. Panel b shows the results (except for SCARED, which lacks overall precision) separately by age (Panel a), gender (Panel b), living space per school-age child (Panel c) and school track (panel d). Extended Data Table 1 shows the detailed regression results (including the results for SCARED). 


\section{Not everyone suffered equally}

The strain and the ability to shoulder the burden imposed by school closures likely varied with individual maturity and living conditions (see Figure $2 \mathrm{~b}$ as well as Extended Data Table 3 ). Subgroup analysis reveals that younger children struggled most with the strain caused by school closures, with the effects declining monotonically with age (Figure $2 \mathrm{~b}$, Panel a). The youngest in our sample, the 11-year-olds, experienced drastic losses in HRQoL ($0.213 \mathrm{sd}, \mathrm{P}<0.001)$ and an increase in psychosomatic symptoms $(0.210 \mathrm{sd}, \mathrm{P}<0.01)$ as well as in behavioral and emotional problems $(0.240 \mathrm{sd}, \mathrm{P}<0.001)$ and in depressive symptoms $(0.146 \mathrm{sd}, \mathrm{P}<0.05)$. Effects quickly fade out, loosing precision by mid adolescence, from age 15-years-old onwards.

Boys coped much worse with the situation than girls (Figure $2 b$, Panel $b$ ). This is visible in a significant drop in HRQoL $(-0.161 \mathrm{sd}, \mathrm{P}<0.001)$ and an increase in psychosomatic symptoms $(0.094 \mathrm{sd}, \mathrm{P}<0.05)$, behavioral and emotional problems $(0.116 \mathrm{sd}, \mathrm{P}<0.01)$ and depressive symptoms $(0.104 \mathrm{sd}, \mathrm{P}<0.05)$. Girls did not suffer any deterioration in mental health.

To proxy the situation at home, we analyze the effects separately by the living space available per schoolaged child (Figure $2 \mathrm{~b}$, Panel c). In homes with limited living space (below the median), adolescents suffered most from the burden imposed by the school closure, visible in a deterioration of their HRQoL (KIDSCREEN-10: -0.182 $\mathrm{sd}, \mathrm{P}<0.001$ ) and an increase in symptoms of mental health problems (HBSC-SCL: $0.097 \mathrm{sd}, \mathrm{P}<0.05 ; \mathrm{SDQ}: 0.102$ $\mathrm{sd}, \mathrm{P}<0.05 ; \mathrm{CES}-\mathrm{DC}: 0.107 \mathrm{sd}, \mathrm{P}<0.05)$ ). In homes with more generous living space (above the median), adolescents did not experience a deterioration in their mental health.

\section{School closures a driver of the mental health crisis}

To judge about the contribution of school closures to the mental health crisis, we compare our results to the prepandemic mental health levels as reported in the nationwide, representative BELLA cohort study 2017 $\left(n=1^{\prime} 556\right)$. Considering the precision of the estimates shown in Figure $2 a$, we deliberately restrict this comparison to youth HRQoL. Figure 3 shows the changes in youth HRQoL, measured in sd changes of youth prepandemic HRQoL (as reported in BELLA 2017).

Corroborating previous results, we document a sizeable drop in youth HRQoL already at the end of the first lockdown (by $-0.692 \mathrm{sd}$, with the $95 \%$ confidence interval ranging from $-0.633 \mathrm{sd}$ to $-0.752 \mathrm{sd})^{20,21,30}$. The impact of prolonged school closures is sizeable in comparison to the overall drop in HRQoL. Figure 3 displays the effect of four additional weeks school closure, and thus of the average prolonged period of school closures experienced by the adolescents in our sample. We abstain from making any predictions for longer periods of school closure, as this would rely on out-of-sample predictions and thus, require strong assumptions. For our sample, we observe a reduction in HRQoL by $-0.430 \mathrm{sd}$ ( $95 \%$ confidence interval: $[-0.193 \mathrm{sd} ;-0.667 \mathrm{sd}]$ ). This effect corresponds to 62.1\% (or between $27.9 \%$ and $96.4 \%$ with $95 \%$ certainty) of the overall drop in HRQoL observed over the first lockdown period. Alarmingly, there is no recovery in youth mental health over the course of the pandemic. In winter 2020/21, when schools were largely closed across the German federal states, youth HRQoL dropped even further to -0.811 sd ( $95 \%$ confidence interval: [-0.749 sd; $-0.872 \mathrm{sd}$ ]) below pre-pandemic levels, and in fall 2021 , when vaccines were widely available for adolescents, youth HRQoL still lingered -0.544 sd $(95 \%$ confidence interval: [-0.483 sd to $-0.604 \mathrm{sd}]$ ) below pre-pandemic levels.

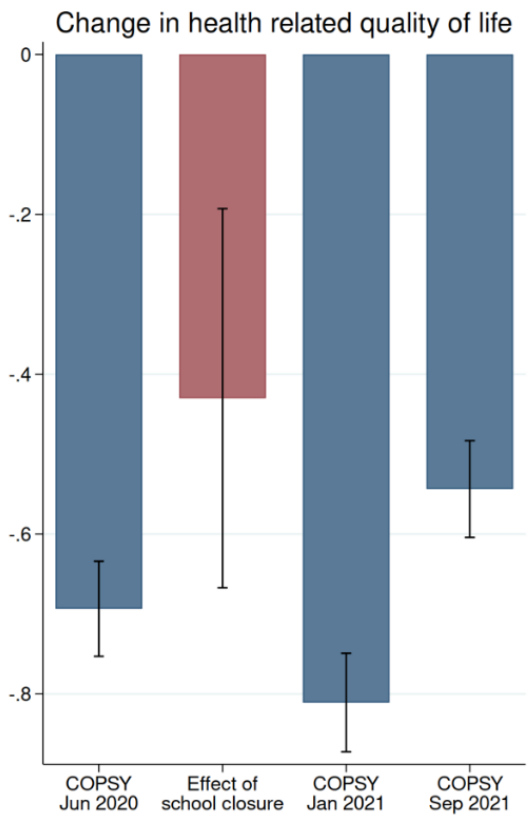

Figure 3: Contribution of school closures to the youth mental health crisis: The blue bars display the relative change (measured in standard deviations) in adolescents' HRQoL in COPSY Wave 1 (May 26 - June 10, 2020), COPSY Wave 2 (December 17, 2020 - January 25, 2021) and COPSY Wave 3 (September 14 - October 11,2021 ) compared to pre-pandemic levels (as in BELLA 2017), respectively. The red bar shows the estimated deterioration in HRQoL due to school closures during the first lock down using data from COPSY Wave 1. The effect is calculated for the average duration of 3.95 additional weeks of school closure used for estimating the causal effect of school closures. The black bars indicate the $95 \%$ confidence interval. 


\section{Spike in helpline calls because of family problems}

It is important to bear in mind that school closures did not happen in isolation. The additional containment measures, such as contact reductions or home-office, left little room for families or friends to buffer adolescents' lack of daily routine, educational guidance by teachers, and social interactions. To shed some light on the issues and concerns preoccupying adolescents during the pandemic, and in particular when affected by school closures, we draw upon high-frequency and real-time data from the largest and most frequented crisis helpline for children and adolescents in Germany ("Nummer gegen Kummer", in particular the "Kinder- und Jugendtelefon").

Figure 4a displays the development of call volumes over the course of the pandemic relative to the call volume in 2019 . We focus on calls related to problems with the family (which accounted for $20.5 \%$ of the overall call volume prior to the pandemic, or to be more precise in 2019) and with friends (14.6\% of the overall call volume in 2019 when referring to peer problems in general, and 4.4\% when focusing on friendship problems in particular) as well as because of fear or insecurity (4.4\% in 2019). The main take-aways from Figure $4 a$ are as follows: i) after relatively little movement over the course of 2019 , calls due to general fear or insecurity spiked with the outbreak of the pandemic ( $80.0 \%$ above the pre-pandemic level) and followed closely the trend in case rates (spiking in winter 2020/2021 at $95.9 \%$ above the pre-pandemic level and going down to the pre-pandemic level in summer 2021, when vaccines were largely available for adolescents); ii) turning to calls because of family problems, we observe a steady increase in calls (up to $40.6 \%$ in comparison to the pre-pandemic level) after the outbreak of the pandemic, peaking at 54.9\% above pre-pandemic level in winter 2020/21 and remaining above pre-pandemic levels until the end of 2021; iii) calls because of problems with friends shortly dipped after the outbreak of the pandemic to steadily increase over the first wave of school closure (peaking at $47.4 \%$ above prepandemic levels shortly thereafter), reaching its second peak during the second wave of school closure in winter 2020/21 (up to $46.4 \%$ above the pre-pandemic level), but quickly recovering whenever schools reopened.

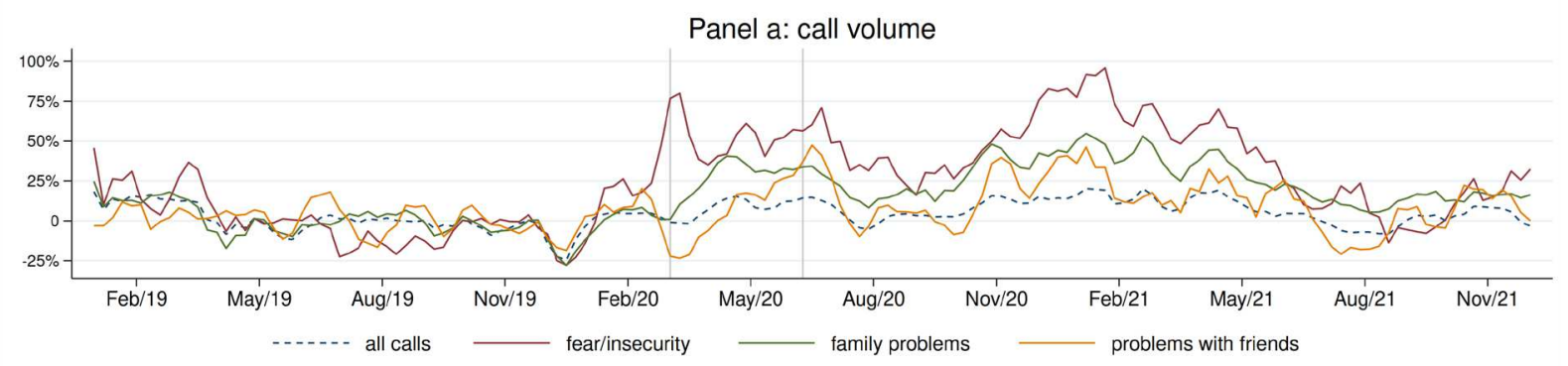

Panel b: the effect of one additional week of school closure on call volume
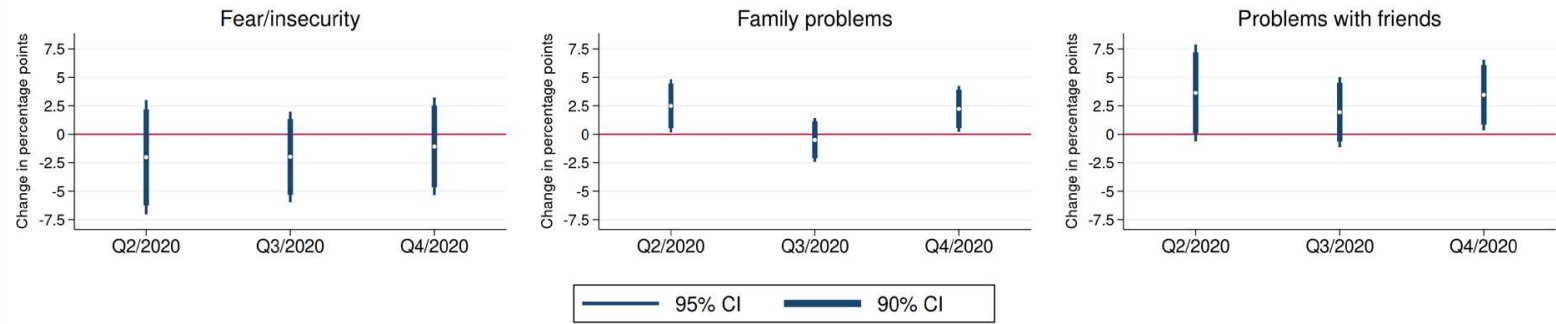

Figure 4a,b: Helpline calls before and during the pandemic: Panel a shows the call volume as a 5 -weeks moving average. The call volume is
calculated as either the total duration of all calls or the total duration of all calls mentioning a particular topic. Each call volume is standardized such that it has a mean of 0 in 2019. Panel b shows the causal effect of one additional week of school closure on call volume of a particular topic in the respective quarter of 2020. The effect size is measured in percentage points change relative to its 2019 level.

Figure $4 \mathrm{~b}$ displays the relative (to the pre-pandemic level) increase in the call volume caused by school closures, experienced by adolescents in the second quarter 2020. We report effects in the short- (second quarter 2020), medium- (third quarter 2020) and long-run (fourth quarter 2020). We abstain from reporting even longer-run effects, as the second phase of nationwide school closures started right after the Christmas holidays 2020/21, rendering an analysis of the mental health effects caused by the initial school closures going beyond 2020.

Reassuringly, we do not observe any causal relationship between the duration of school closure and adolescents' fear or insecurity. This null result raises confidence in our empirical strategy capturing the overall effect of the pandemic and other pandemic measures. However, family problems increase because of school closures. One additional week of school closure experienced in the second quarter 2020 caused an immediate increase in the call volume by 2.5 percentage points (henceforth $p p, p<0.05$ ), followed by a subsequent recovery phase with no excess calls from adolescents who had suffered under prolonged school closure, yet to spike again with the skyrocketing case rates and the threat of repeated school closures towards the end of 2020 (by 2.2 pp, 
$p<0.05$, for each additional week of school closure experienced during the second quarter 2020). Turning to problems with friends, we see a similar pattern with school closures causing an immediate increase in helpline calls because of problems with friends (by $3.6 \mathrm{pp}$ per additional week, $\mathrm{p}<0.1$ ), a recovery during the second quarter, yet to increase again during the fourth quarter (by $3.4 \mathrm{pp}, \mathrm{p}<0.05$ ).

\section{Discussion}

We relied on variation in school reopening strategies across the German federal states to identify the causal impact of school closures on youth mental health. Drawing upon nationwide, population-based survey data on adolescents' mental health before and during the COVID-19 pandemic allowed us to determine and compare the mental health impact of the pandemic, and in particular, of one of the most heavily debated pandemic measures on youth mental health. We showed that school closures significantly contributed to the recent increase in youth mental health. On average, school closures explain around two thirds of the increase in youth mental health problems over the first pandemic wave. Using data from crisis helpline data allowed us to shed light on the problems preoccupying adolescents during the pandemic, mostly struggling with family problems and with a lack of support experienced by friends. Noteworthy, the school closures significantly worsened this problems.

Our results likely reflect only the tip of the iceberg for several reasons. On the one hand, our analysis was limited to self-reports on $\mathrm{HRQoL}$ and answers to various screening questionnaires for mental health problems. Considering that the screening instruments act as early warning systems for severe mental health issues, there is certainly a need to dig deeper and to use medical reports or health insurance claim data to analyze actual diagnoses. On the other hand, we estimated the effects of the initial school closures only, lasting between 4.7 and 13 weeks. In Germany, 25 or more additional weeks of school closure followed, in the U.S., students were exempted from in-person learning for 71 weeks in total ${ }^{14}$. The effects may obviously not accumulate week by week. Many adolescents may have adjusted to and learned to live with the new situation. Yet, the pandemic and its related measures represent a continuous burden for adolescents and their families, depriving adolescents from the engaging, protective family environment and the positive social interactions urgently needed for a swift recovery to happen.

With these results, we added one piece to the puzzle needed to conduct a cost-benefit analysis of the pandemic measures. We interpret our results as a call for caution when considering school closures as a measure to stop the viral spread. Our results should also be seen as a call for action to address the needs of adolescents struggling with the negative consequences of school closures, implying disturbances to their daily routines and educational guidance as well as a disruption in social contacts and interactions.

\section{References}

[1] AAP-AACAP-CHA Declaration of a National Emergency in Child and Adolescent Mental Health. https://www.aap.org/en/advocacy/child-and-adolescent-healthy-mental-development/aap-aacap-cha-

declaration-of-a-national-emergency-in-child-and-adolescent-mental-health/ (2021).

[2] Holmes, E.A. et al. Multidisciplinary research priorities for the COVID-19 pandemic: a call for action for mental health science. Lancet Psychiatry 7, 547-560 (2020).

[3] Protecting Youth Mental Health: The U.S. Surgeon General's Advisory. https://www.hhs.gov/sites/default/files/surgeon-general-youth-mental-health-advisory.pdf (2021).

[4] Lee, J. Mental health effects of school closures during COVID-19. Lancet Child \& Adolesc. Health 4, 421 (2020). [5] Engzell, P., Frey, A. \& Verhagen, M.D. Learning loss due to school closures during the COVID-19 pandemic. Proc. Natl. Acad. Sci. 118, e2022376118 (2021).

[6] Brülhart, M., Klotzbücher, V., Lalive, R. \& Reich, S.K. Mental health concerns during the COVID-19 pandemic as revealed by helpline calls. Nature 600, 121-126 (2021).

[7] Pierce, M. et al. Mental health before and during the COVID-19 pandemic: a longitudinal probability sample survey of the UK population. Lancet Psychiatry 7, 883-892 (2020).

[8] Tanaka, T. \& Okamoto, S. Increase in suicide following an initial decline during the COVID-19 pandemic in Japan. Nat. Hum. Behav. 5, 229-238 (2021).

[9] Bagattini, A. Children's well-being and vulnerability. Ethics Soc. Welf. 13, 211-215 (2019).

[10] Brown, B.B. Adolescents' Relationships with Peers. in Handbook of Adolescent Psychology (eds. Lerner, R.M. \& Steinberg, L.) 363-394 (John Wiley \& Sons Inc., 2004).

[11] De Goede, I.H.A., Branje, S.J.T. \& Meeus, W.H.J. Developmental changes in adolescents' perceptions of relationships with their parents. J. Youth Adolesc. 38, 75-88 (2009).

[12]Editorial Note. Young people's mental health is finally getting the attention it needs. Nature 598, 235-236 (2021).

[13] Racine, N. et al. Global prevalence of depressive and anxiety symptoms in children and adolescents during COVID-19: a meta-analysis. JAMA Pediatr. 175, 1142-1150 (2021). 
[14] UNESCO. Global monitoring of school closures caused by COVID-19. https://en. unesco.org/covid19/educationresponse (2021).

[15] Hemmeter, M.L., Ostrosky, M. \& Fox, L. Social and emotional foundations for early learning: a conceptual model for intervention. School Psych. Rev. 35, 583-601 (2006).

[16] Dong, E., Du, H. \& Gardner, L. An interactive web-based dashboard to track COVID-19 in real time. Lancet Infect. Dis. 20, 533-534 (2020).

[17] Vornberger, J. The impact of school closures on child and adolescent mental health. mimeo (2020).

[18] Wooldridge, J. Two-way fixed effects, the two-way mundlak regression, and difference-in-differences estimators. SSRN 3906345, (2021).

[19] Angrist, J.D. \& Pischke J.S. Mostly Harmless Econometrics: An Empiricistís Companion. (Princeton University Press, 2009)

[20] Ravens-Sieberer, U. et al. Quality of life and mental health in children and adolescents during the first year of the COVID-19 pandemic: results of a two-wave nationwide population-based study. Eur. Child Adolesc. Psychiatry, 1-14. (2021).

[21] Ravens-Sieberer, U. et al. Impact of the COVID-19 pandemic on quality of life and mental health in children and adolescents in Germany. Eur. Child Adolesc. Psychiatry, 1-11 (2021).

[22] Ravens-Sieberer, U. et al. The Kidscreen Questionnaires: Quality of Life Questionnaires for Children and Adolescents - handbook. (Pabst Science Publishers, 2006).

[23] Haugland, S., Wold, B., Stevenson, J., Aaroe, L.E. \& Woynarowska, B. Subjective health complaints in adolescence: a cross-national comparison of prevalence and dimensionality. Eur. J. Public Health 11, 4-10 (2001).

[24] Goodman, R. The strengths and difficulties questionnaire: a research note. J. Child Psychol. Psychiatry 38, 581-586 (1997).

[25] Barkmann, C., Erhart M. \& Schulte-Markwort, M. The German version of the centre for epidemiological studies depression scale for children: psychometric evaluation in a population-based survey of 7 to 17 years old children and adolescents-results of the BELLA study. Eur. Child Adolesc. Psychiatry 17, 116-124 (2008).

[26] Birmaher, B. Psychometric properties of the screen for child anxiety related emotional disorders (SCARED): a replication study. J. Am. Acad. Child Adolesc. Psychiatry 38, 1230-1236 (1999).

[27] Kurth, B.M. The RKI health monitoring - what it contains and how it can be used. Public Health Forum 20, 4 (2012).

[28] Ravens-Sieberer, U. et al. The longitudinal BELLA study: design, methods and first results on the course of mental health problems. Eur. Child Adolesc. Psychiatry 24, 651-663 (2015).

[29] Otto, C. et al. Mental health and well-being from childhood to adulthood: design, methods and results of the 11-year follow-up of the BELLA study. Eur. Child Adolesc. Psychiatry 30, 1559-1577 (2021).

[30] Ravens-Sieberer, U. et al. Seelische Gesundheit und psychische Belastungen von Kindern und Jugendlichen in der ersten Welle der COVID-19-Pandemie-Ergebnisse der COPSY-Studie. BundesgesundheitsblattGesundheitsforschung-Gesundheitsschutz 64, 1512-1521 (2021).

[31] Lange, M. et al. KiGGS wave 2 longitudinal component - data collection design and developments in the number of participants in the KiGGS cohort. Journal of Health Monitoring 3, 92-107 (2018).

[32] Krause, K.R. et al. International consensus on a standard set of outcome measures for child and youth anxiety, depres- sion, obsessive-compulsive disorder, and post-traumatic stress disorder. Lancet Psychiatry 8 , 76-86 (2021).

[33] Ravens-Sieberer, U. et al. The European KIDSCREEN approach to measure quality of life and well-being in children: development, current application, and future advances. Qual. Life Res. 23, 791-803 (2014).

[34] Radloff, L.S. The CES-D scale: a self-report depression scale for research in the general population. Appl. Psychol. Meas. 1, 385-401 (1977).

[35] Weitkamp, K., Romer, G., Rosenthal, S., Wiegand-Grefe, S. \& Daniels, J. German screen for child anxiety related emotional disorders (SCARED): reliability, validity, and cross-informant agreement in a clinical sample. Child Adolesc. Psychiatry Ment. Health 4, 1-8 (2010).

[36] Correia, S. Linear models with high-dimensional fixed effects: An efficient and feasible estimator. Tech. rep. Working Paper (2016).

[37] Abadie, A., Athey, S., Imbens, G.W. \& Wooldridge, J. When should you adjust standard errors for clustering? NBER Work Pap Ser., 1-28 (2017).

[38] Eiser, C. \& Morse, R. Can parents rate their child's health-related quality of life? Results of a systematic review. Qual. Life Res. 10, 347-357 (2001).

[39] Upton, P., Lawford, J. \& Eiser, C. Parent-child agreement across child health-related quality of life instruments: a review of the literature. Qual. Life Res. 17, 895-913 (2008). 


\section{Methods:}

\section{Data on School Closures}

In the absence of a comparative and transparent overview, we processed all state-specific Corona protection ordinances and compiled a dataset on the state-specific school closure and re-opening strategies ${ }^{17}$. Each federal state enjoyed educational sovereignty and thus, decided independently about the school closure and particularly the reopening strategy. Decisions were taken for each school track and grade level separately. Thus, the resulting dataset has entries by state, school track (which, for simplicity, are restricted to the main primary and secondary school forms) and grade levels. We assigned the corresponding start and end dates (defined as soon as a partial re-opening took place) of school closures to each cell defined by a unique combination of state, school track, and grade level. The number of weeks between the start and the end dates define the duration of school closure by state and school track specific grade level (see Extended Data Figure 1 for the resulting variation by state and grade-level differentiated by school track and limited to the variation used for estimations). The varying strategies adopted by the 16 German federal states lead to differences in the timing of closure and re-opening, and as such in the duration of school closure. Overall, priority was given to the graduating students (whose grade differ by state and school track, see Extended Data Figure 1 for an overview) who started to return already after 4.7 weeks. and in total a substantial variation within grade levels and within school tracks ranging from 4.7 to 13 weeks occurs.

\section{Nationwide data on COvid-19 and PSYchological Health (COPSY)}

We rely on data from the unique, nationwide, population-based German COPSY study (COVID-19 and Psychological Health) with three survey waves during the pandemic ${ }^{20}$. COPSY builds upon the nationwide, longitudinal, population-based BELLA cohort study (Behavior and Wellbeing of Children and Adolescents in Germany) ( $n=1^{\prime} 556$ ), which is the mental health module of the German National Health Interview and Examination Survey for Children and Adolescents (KIGGS) ${ }^{29}, 31$. As such, we can compare and anchor effects of the COVID-19 pandemic and related policy measures to the pre-pandemic mental health status of children and adolescents $^{9,12,13,14,15}$. COPSY was conducted online among $n=1$ '040 11-17-year-old adolescents first between May 26 and June 10, 2020. Follow-up surveys with $n=1^{\prime} 077$ (response rate $=85.1 \%$ plus sample increase) and $\mathrm{n}=1^{\prime} 139$ (response rate $=73.7 \%$ plus sample increase) adolescents took place between December 17,2020 , and January 25, 2021 (September 14, and October 11, 2021). In addition, $n=1^{\prime} 586\left(n=1^{\prime} 625, n=1^{\prime} 618\right)$ parents of 717-year-old children and adolescents participated in the (follow-up) surveys. Since our identification strategy, described below, only allow us to estimate causal effects of the short-run mental health effects of school closure, we focus on survey wave 1 and use survey waves 2 and 3 to provide an outlook on the development of adolescents' mental health during the COVID-19 pandemic.

To measure mental health, we rely on internationally accepted, validated, and comparable measures that are in accordance with the guidelines of the International Consortium for Health Outcomes Measurement ${ }^{21,32}$. Specifically, we use the KIDSCREEN-10 Index and the HBSC Symptom Checklist to measure adolescents' wellbeing and psychosomatic complaints. The KIDSCREEN-10 Index is based on the KIDSCREEN-52 and constitutes a global measure for health-related quality of life (HRQoL). It is computed using the responses on a five-point Likert scale (from "never" to "always", or "not at all" to "extremely") with ten questions capturing information e.g., "Have you felt fit and well during the previous week?". The KIDSCREEN-10 Index is developed according to the item response theory (international T-values based on RASCH modeling) ${ }^{22,}{ }^{33}$. The HBSC Symptom Checklist (HBSC-SCL) contains eight questions assessing the frequency of psychosomatic complaints (e.g., headache, nervousness) within the past week on a five-point response scale (from "not at all" to "daily") ${ }^{23}$. We draw upon three clinical scales. The Strengths and Difficulties Questionnaire (SDQ) provides information about emotions, behaviors, and relationships regarding children and adolescents during the previous week. It contains in total 20 items divided in four subscales on emotional (e.g., "Many worries, often seems worried"), conduct (e.g., "Often lies or cheats"), hyperactivity (e.g., "Constantly fidgeting or squirming"), and peer problems (e.g., "Often fights with other children or bullies them"), each providing three response options from "not true" to "certainly true" 24 . We further used the Center for Epidemiological Studies Depression Scale for Children (CES-DC) to examine depressive symptoms. This scale is generated based on seven items (e.g., "I felt sad") whose frequency during the previous week is scored on a scale of 0 (= "not at all") to 3 ("a lot") ${ }^{25,34}$. Finally, we relied on the nine-item generalized anxiety subscale of the German Screen for Child Anxiety Related Emotional Disorders (SCARED). Here, adolescents are asked to score statements such as "I am nervous" with three response options ( $0=$ "not true or hardly ever true" to 2 = "very true or often true") ${ }^{26,35}$. All scales stem from the youth survey except the $\mathrm{SDQ}$, which comes from the parental questionnaire. 


\section{Merged dataset (COPSY wave 1 and school closure data)}

We can merge the self-compiled data set on the weeks of school closure with the COPSY data via adolescents' state, grade level, and school track. The resulting sample contains in $n=907$ 11-17-year-old adolescents (age mean: 13.8; sd:1.9; see Table Extended Data Table 1, representative for German youth (132 observations are dropped because of missing information on the state and/or school track). Interviews for COPSY took place while some of the adolescents were still at home. Since we lack the exact survey date, we take the start date of COPSY, May 26,2020, to impute a conservative measure of individual duration of school closure for these cases. Using this imputation method, adolescents in our sample experience school closure lasting at least 4.7 and at most 10.1 weeks. Using an alternative imputation method, the end date of COPSY, June 10, 2020, to define the individual duration of school closures, results in a maximum duration of 12.3 weeks.

Our sample includes 468 girls and 439 boys. Of these, $20.0 \%$ (sd: 0.4 ) attend a comprehensive school comprising both primary and secondary school, $39.4 \%$ (sd: 0.5 ) attend the vocational track of secondary school, and $40.6 \%$ (sd: 0.5 ) the academic track of secondary school. On average, the families have 1.6 children (sd: 0.7 ) and a living space of $133.0 \mathrm{sqm}$ (sd: 55.3). Our outcomes measures KIDSCREEN-10 (mean: 45.2; sd: 8.0), HBSCSCL (mean: -36.0; sd: 4.5), SDQ (mean: 9.0; sd: 5.4), CES-DC (mean: 11.3; sd: 3.6) and SCARED (mean: 5.6; sd: 4.2), are all standardized to have mean 0 and $\mathrm{sd} 1$.

\section{Causal analysis of the impact of school closures}

We employ a difference-in-differences method, or more formally a linear regression model with two levels of fixed effects ${ }^{18,19,36}$, to identify the effect of school closures on adolescents' mental health. The variable of interest, the individual duration of school closures, is perfectly determined by the combination of the individual state and the school track-specific grade level. The difference-in-differences method accounts for two levels of fixed effects, a set of state and school track-specific grade level fixed effects, and thus absorbs any level differences between states and school track-specific grade levels in adolescents' mental health. Identification is thus based on the remaining variation in the duration of school closures within the states (across school trackspecific grade levels) and within school track-specific grade levels (across states). The identifying assumption is thus that there are no systematic, confounding factors driving the deviation in adolescents' mental health from the mental health predicted for any adolescents residing in state $s$ and attending the school track-specific grade level xc, other than the duration of school closure.

More formally, we model youth mental health using the following equation:

$$
Y_{i s c x}=\beta D_{s c x}+\gamma_{s}+\gamma_{c x}+\delta X_{i}+\epsilon_{i s c x}
$$

where $Y_{i s c x}$ constitutes the dependent variable, comprising the different measures of the mental health of individual $i$ that attends school track $\mathrm{x}$ in grade $\mathrm{c}$ in state $\mathrm{s}$. We standardize all outcome variables to a mean 0 and sd 1, facilitating the comparison across different mental health dimensions and the interpretation of the effect size. The independent variable $D_{s c x}$ denotes weeks of school closure. We further control for adolescents' age (in years) and gender (using a dummy $=1$ if female) summarized by the vector $X_{i}$. We include state $\left(\gamma_{s}\right)$ and school track-specific grade level $\left(\gamma_{c x}\right)$ fixed effects. $\epsilon_{i s c x}$ represents an idiosyncratic error term. All estimates shown in the paper (specifically in Figure 2a and Extended Data Table 2, Panel a) result from estimation equation (1) using ordinary least square and clustering the standard errors at the state*school track-specific grade level, and thus on treatment level ${ }^{37}$.

\section{Sensitivity analysis}

To check the sensitivity of our baseline results, we run a series of alternative specifications presented in Extended Data Table 2, Panel b-h. We first list the baseline results (Extended Data Table 2, Panel a). The remaining panels show the estimates resulting after modifying one component of baseline specification (equation 1). First, we use a more parsimonious approach and exclude all individual control variables contained in the vector $X_{i}$ (Panel b). In Panel c we aim at absorbing any level differences in adolescents' mental health across school tracks within states and thus control for a fully interacted set of state and school track fixed effects $\gamma_{s x}$ (instead of a set of state fixed effects $\gamma_{S}$ only). In Panel d we include the second order polynomial of length of school closure $D_{S C x}^{2}$ as a further covariate in equation (2) to allow for any non-linear effects. In the robustness checks in Panel e and $\mathrm{f}$, we reconsider the imputation of the duration of school closures $D_{s c x}$. In Panel e we use the survey end date (instead of the survey start date) to impute the duration of school closure for all adolescents that had not returned to school prior to the May 26, 2020 (the start of COPSY Wave 1). In Panel f, we adjust the duration of school closure for any school holidays taking place during the lockdown. Here, we subtract the length of vacations from the duration of school closure and use it as the main explanatory variable. In Panel g, we use individual 
reports about the school situation in the week prior to the survey date instead of the predicted duration of school closure. Specifically, we rely on parental reports on whether their adolescent child had returned to school or still lingered in home-schooling. For this sensitivity check, we rely on a dummy variable taking the value 1 if teaching takes place mainly or exclusively at home. Following the recommendation to use both self-reported and externally evaluated answers to mental health $\mathrm{scales}^{38,39}$, we re-estimate equation (1) using parental reports on adolescents' mental health as the dependent variable $Y_{\text {iscx }}$ (see Panel h). We can do so only for the KIDSCREEN10 index and HBSC-SCL scale, as the parental questionnaire does not contain the further screening devices for mental health problems. For comparability, we restrict the sample to parents reporting on their adolescent children only (age interval 11-17).

\section{Subgroup analysis}

For the subgroup analysis in Figure $2 \mathrm{~b}$ and Extended Data Table 3, we adapt the baseline model of equation (2). Specifically, we adjust the main explanatory variable for each subgroup analysis. To examine the effect of school closures by adolescents' age (shown in Fig. $2 \mathrm{~b}$ and Extended Data Table 3, Panel a), we add the interaction terms between weeks of school closure $D_{s c x}$ and a full set of age dummies ( $a_{i j}$, which equals 1 if the individual $\mathrm{i}$ is aged $\mathrm{j}$, and $=0$ otherwise). The main effect of age is included in the vector $X_{i}$. This model (see equation 3 ) allows us to measure the effect $\beta_{j}$ of weeks of school closure on mental health for each age group j separately.

$$
Y_{i s c x}=\sum_{j=11}^{17}\left(\beta_{j}\left(D_{s c x} * a_{i j}\right)+\gamma_{s}+\gamma_{c x}+\delta X_{i}+\epsilon_{i s c x}\right)
$$

In Panel $b$ of Figure $2 b$ and Extended Data Table 3, Panel b, we present the effect on mental health by adolescents' gender. For this, we include the interaction of weeks of school closure $D_{s c x}$ and the female dummy $f_{i}$ as well as the interaction of weeks of school closure $D_{s c x}$ and the male dummy $m_{i}$ (see equation (4)). This allows us to identify the effect of weeks of school closure on mental health $Y_{i s c x}$ for boys $\left(\beta_{m}\right)$ and girls $\left(\beta_{f}\right)$ separately.

$$
Y_{i s c x}=\beta_{f}\left(D_{s c x} * f_{i}\right)+\beta_{m}\left(D_{s c x} * m_{i}\right)+\gamma_{s}+\gamma_{c x}+\delta X_{i}+\epsilon_{i s c x}
$$

Finally, we show the effect by living space per school-aged child (see Figure $2 \mathrm{~b}$ and Extended Data Table 3, Panel c). For this purpose, we introduce an interaction term between weeks of school closure $D_{s c x}$ and a dummy variable indicating that individual $i$ resides in a living space smaller than the median of 85 square meters (captured $l_{i}$, which equals 1 if the living space is smaller than the median living space, and 0 otherwise). Moreover, we add an interaction term between weeks of school closure $D_{s c x}$ and a dummy variable $h_{i}$ which equals 1 if individual $i$ resides in a living space larger than the median of 85 square meters, and 0 otherwise. We add the dummy variable $l_{i}$ as further control variable and estimate equation (5), as follows

$$
Y_{i s c x}=\beta_{l}\left(D_{s c x} * l_{i}\right)+\beta_{h}\left(D_{s c x} * h_{i}\right)+\gamma_{s}+\gamma_{c x}+\delta X_{i}+\rho l_{i}+\epsilon_{i s c x}
$$

\section{Helpline data}

For the analysis in Figure 4, we rely on data from the largest and most frequented German crisis helpline with a focus on children and adolescents ("Nummer gegen Kummer", in particular the "Kinder- und Jugendtelefon"). The helpline exists since 1980 and currently consists of 76 centers operating across Germany. The service is free of charge and can be reached from Monday to Saturday between $2 \mathrm{pm}$ and $8 \mathrm{pm}$. Further information is available online at www.nummergegenkummer.de. The helpline guarantees anonymity to their callers, and it is impossible to identify callers from conversation-level data we have at hand. Yet, callers are informed that anonymous call data are collected for reporting and statistical purposes, explicitly in the terms and conditions, and implicitly in annual reports and online publications.

Information on detailed, non-exclusive conversation topics allows us to track the importance of problems among the vulnerable population of callers. Counselors report the age of callers if stated during the conversation, or provide an estimate, allowing us to approximate the most likely grade level for each caller. Together with information on the location of the receiving helpline center, we link the call data with our data on school closure by the federal state and the approximated grade level. Since we do not possess information on the school track, we use the longest school closure for the respective grade level. Obviously, the matching procedure is substantially less precise than the procedure for the survey data (given imprecision in the state, the grade level and the lack of the school track). In addition, calls from mobile phones are not necessarily connected within a state, and as no center operates in the state of Thuringia, calls originating there are answered in neighboring or 
other states. Overall, the data includes information on 282,688 conversations for the time from January 2019 to December 2021 . We focus on the 179,568 calls by callers of age 11 to 17 to increase the comparability with previous analyses using the survey data; estimation results are based on 126,006 calls received up to December 2021.

In Figure 4a, we show the development of call volumes over time. Call volume is defined as the total duration of conversations per week (measured in minutes), normalized to reflect the relative difference (in percent) to the overall mean in 2019. We focus on three categories of conversation topics/caller issues: fear/insecurity, family problems, and problems with friends. Note that these categories are non-exclusive, as multiple topics can be reported for each conversation.

Figure $4 \mathrm{~b}$ shows the estimate of the causal effect of prolonged school closure. Here we aggregate weekly call volumes by state and predicted grade level for the years 2019 and 2020 and estimate the dynamic relationship in a panel estimation framework as illustrated in equation (6).

$$
Y_{s c t}=\sum_{i=2}^{4} \beta_{q i} 1_{q i} \mathrm{D}_{\mathrm{sct}}+\gamma_{s}+\gamma_{c}+\gamma_{t}+\gamma_{p}+\epsilon_{s c t}
$$

where $Y_{s c t}$ denotes the call volume (standardized to mean 0 in 2019) related to topic $Y$ for state s and grade level c in week t. We create an indicator variable $1_{q 2}$ that is one for week 14 to 26 of 2020 and 0 otherwise. Similarly, the indicator variables $1_{q 3}$ and $1_{q 4}$ are one for week 27 to 39 and for week 40 to 52 and zero otherwise, respectively. We interact these indicator variables with our measure of predicted weeks of school closure $D_{s c t}$ experienced by grade level c in state s. This implies that $D_{s c t}$ may vary week by week in the second quarter of 2020 and but remains constant for each grade level state combination in the third and fourth quarter of 2020 (since the end of the first period of school closures were last on June 15, 2020). Thus, $\beta_{q i}$ can be interpreted as the impact of an additional week of school closure experienced during the first lockdown (in the second quarter) on the call volume in the second, third, and fourth quarter of 2020, respectively. The magnitude of $\beta_{q i}$ is the change in percentage points over the respective level in 2019 for each week of school closure.

To grant causal identification (see discussion above), we account for state and grade level fixed effects by $\gamma_{s}$ and $\gamma_{c}$ as well as for seasonal patterns by $\gamma_{t}$. Further, we account for an overall increase in call volume due to the pandemic itself by including $\gamma_{p}$. This is a dummy variable that is 1 for each week since March 16, 2020, the week all schools closed, and 0 for every week before. $\epsilon_{s c t}$ is the error term and we use clustered robust standard errors and cluster them on age* state* quarter*year level to allow for arbitrary autocorrelation within the respective group. 

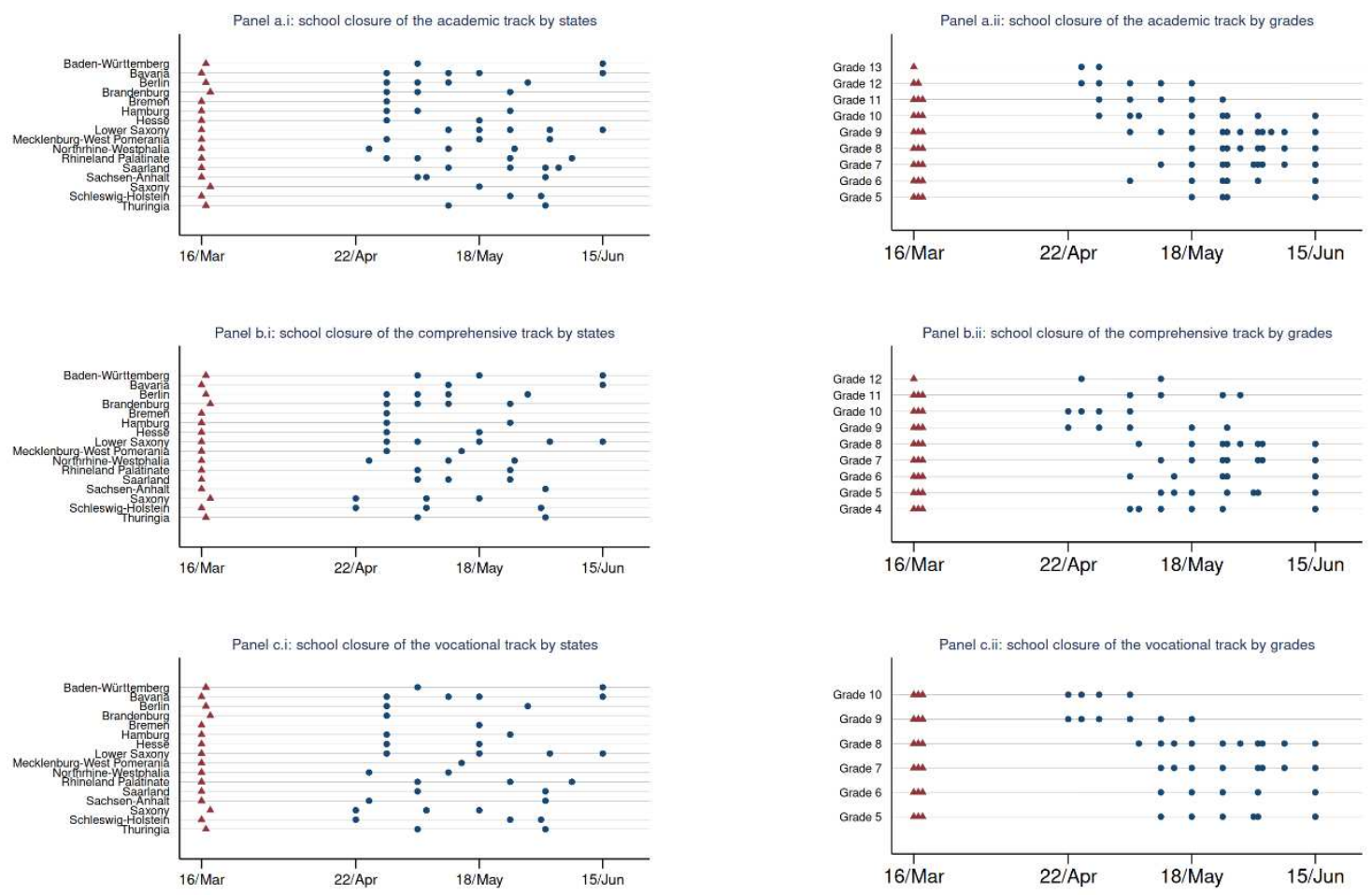

$$
\text { - Closure Partial reopening }
$$

Extended Data Figure 1: Variation in school closure and re-opening strategies by (i) states and (ii) grade levels, separately for the academic track (Panel a), the comprehensive track (subsuming various types of primary and secondary schools) (Panel b), and the vocational track (Panel c). Data are compiled by screening the statespecific Corona protection ordinances. Each blue dot represents the date when the respective federal government (partially) re-opened schools for selected grade levels (Paneli) and the resulting variation in the reopening dates by grade-levels across the federal states (Panel ii). The variation shown is restricted to the grades attended by the adolescents in our sample. 


\begin{tabular}{|c|c|c|c|c|c|}
\hline & $\mathrm{N}$ & Mean & SD & Min & $\operatorname{Max}$ \\
\hline \multicolumn{6}{|l|}{ Panel a: Outcome variables } \\
\hline \multicolumn{6}{|l|}{ HRQoL } \\
\hline KIDSCREEN-10 & 907 & 45.211 & 7.999 & 22.40 & 83.81 \\
\hline \multicolumn{6}{|c|}{ Screening devices for mental health problems } \\
\hline HBSC-SCL & 907 & -35.981 & 4.547 & -40.00 & -12.00 \\
\hline SDQ & 907 & 9.017 & 5.421 & 0.00 & $32.00)$ \\
\hline CES-DC & 907 & 11.291 & 3.645 & 7.00 & 27.00 \\
\hline SCARED & 907 & 5.612 & 4.228 & 0.00 & 18.00 \\
\hline \multicolumn{6}{|l|}{ Panel b: Treatment variable } \\
\hline Weeks of school closure & 907 & 8.728 & 1.574 & 4.71 & 10.14 \\
\hline \multicolumn{6}{|c|}{ Panel c: Sociodemographic background characteristics } \\
\hline Age (in years) & 907 & 13.845 & 1.928 & 11 & 17 \\
\hline Female $(=1,=0$ otherwise $)$ & 907 & 0.494 & 0.500 & 0 & 1 \\
\hline Number of children in household & 907 & 1.561 & 0.694 & 1 & 5 \\
\hline Living space (in sqm) & 907 & 132.978 & 55.316 & 38 & 450 \\
\hline \multicolumn{6}{|l|}{ School track } \\
\hline Vocational ( $=1,=0$ otherwise $)$ & 907 & 0.394 & 0.489 & 0 & 1 \\
\hline Comprehensive $(=1,=0$ otherwise) & 907 & 0.200 & 0.400 & 0 & 1 \\
\hline Academic $(=1,=0$ otherwise $)$ & 907 & 0.406 & 0.491 & 0 & 1 \\
\hline
\end{tabular}

Extended Data Table 1: Descriptive statistics of our estimation sample Number of observations, mean and standard deviation of the main sociodemographic factors and outcome variables of the weighted final dataset including. Weights are developed based on the current German Microcensus (2018). 


\begin{tabular}{|c|c|c|c|c|c|}
\hline & \multirow{2}{*}{$\begin{array}{c}\text { HRQoL } \\
\frac{(1)}{\text { KS-10 }}\end{array}$} & \multicolumn{4}{|c|}{ Screening devices for mental health problems } \\
\hline & & $\begin{array}{c}(2) \\
\text { HBSC-SCL }\end{array}$ & $\begin{array}{c}(3) \\
\text { SDQ }\end{array}$ & $\begin{array}{c}(4) \\
\text { CES-DC }\end{array}$ & $\begin{array}{c}(5) \\
\text { SCARED }\end{array}$ \\
\hline \multicolumn{6}{|l|}{ Panel a: average effect } \\
\hline Weeks of school closure & $\begin{array}{c}-0.112^{* * *} \\
(0.032)\end{array}$ & $\begin{array}{l}0.071^{* *} \\
(0.034)\end{array}$ & $\begin{array}{l}0.083^{* *} \\
(0.036)\end{array}$ & $\begin{array}{l}0.073^{* *} \\
(0.034)\end{array}$ & $\begin{array}{c}0.029 \\
(0.034)\end{array}$ \\
\hline \multicolumn{6}{|c|}{ Panel b: no control variables (i.e. age and gender) } \\
\hline Weeks of school closure & $\begin{array}{c}-0.109^{* * *} \\
(0.032)\end{array}$ & $\begin{array}{l}0.066^{*} \\
(0.034)\end{array}$ & $\begin{array}{l}0.085^{* *} \\
(0.036)\end{array}$ & $\begin{array}{l}0.068^{* *} \\
(0.034)\end{array}$ & $\begin{array}{c}0.021 \\
(0.034)\end{array}$ \\
\hline \multicolumn{6}{|c|}{ Panel c: school-track $\times$ state and school-track $\times$ grade fixed effects } \\
\hline Weeks of school closure & $\begin{array}{c}-0.104^{* * *} \\
(0.038)\end{array}$ & $\begin{array}{l}0.062^{* *} \\
(0.030)\end{array}$ & $\begin{array}{l}0.095^{* *} \\
(0.044)\end{array}$ & $\begin{array}{c}0.087^{* *} \\
(0.042)\end{array}$ & $\begin{array}{c}0.013 \\
(0.040)\end{array}$ \\
\hline \multicolumn{6}{|c|}{ Panel d: second order polynomial of school closure duration } \\
\hline $\begin{array}{l}\text { Weeks of school closure } \\
(\text { Weeks of school closure })^{2}\end{array}$ & $\begin{array}{l}0.233 \\
(0.241) \\
-0.021 \\
(0.015)\end{array}$ & $\begin{array}{c}-0.389 \\
(0.289) \\
0.028 \\
(0.018)\end{array}$ & $\begin{array}{l}-0.586^{*} \\
(0.335) \\
0.041^{* *} \\
(0.020)\end{array}$ & $\begin{array}{c}-0.193 \\
(0.277) \\
0.016 \\
(0.017)\end{array}$ & $\begin{array}{c}-0.091 \\
(0.247) \\
0.007 \\
(0.015)\end{array}$ \\
\hline \multicolumn{6}{|l|}{ Panel e: survey end as cutoff } \\
\hline Weeks of school closure & $\begin{array}{c}-0.087^{* * *} \\
(0.028)\end{array}$ & $\begin{array}{l}0.049^{*} \\
(0.025)\end{array}$ & $\begin{array}{c}0.084^{* * *} \\
(0.029)\end{array}$ & $\begin{array}{l}0.065^{* *} \\
(0.030)\end{array}$ & $\begin{array}{c}0.014 \\
(0.026)\end{array}$ \\
\hline \multicolumn{6}{|c|}{ Panel f: school closure duration without holidays } \\
\hline Weeks of school closure & $\begin{array}{c}-0.106^{* * *} \\
(0.032)\end{array}$ & $\begin{array}{l}0.065^{*} \\
(0.035)\end{array}$ & $\begin{array}{l}0.078^{* *} \\
(0.037)\end{array}$ & $\begin{array}{l}0.067^{*} \\
(0.035)\end{array}$ & $\begin{array}{c}0.026 \\
(0.035)\end{array}$ \\
\hline \multicolumn{6}{|l|}{ Panel g: home-schooling } \\
\hline Currently no school & $\begin{array}{l}-0.110 \\
(0.103)\end{array}$ & $\begin{array}{l}0.169^{*} \\
(0.098)\end{array}$ & $\begin{array}{c}0.037 \\
(0.097)\end{array}$ & $\begin{array}{c}0.091 \\
(0.113)\end{array}$ & $\begin{array}{l}0.184^{* *} \\
(0.093)\end{array}$ \\
\hline \multicolumn{6}{|c|}{ Panel h: parent questionnaire } \\
\hline Weeks of school closure & $\begin{array}{c}-0.109^{* * *} \\
(0.032)\end{array}$ & $\begin{array}{l}0.064^{*} \\
(0.037)\end{array}$ & & & \\
\hline
\end{tabular}

Extended Data Table 2: Short-run impact of one week school closure on self-reported mental health Estimates (including the standard errors in parenthesis) for the effect of one week school closure on standardized measures (mean 0, std 1) of adolescents' HRQoL and screening devices for clinical mental health issues. The sample consists of $n=907$ 11-17-year-old adolescents participating in the first COPSY wave (May 26 - June 10, 2020). 


\begin{tabular}{|c|c|c|c|c|c|}
\hline & \multirow{2}{*}{$\begin{array}{c}\text { HRQoL } \\
(1) \\
\text { KS-10 }\end{array}$} & \multicolumn{4}{|c|}{ Screening devices for mental health problems } \\
\hline & & $\begin{array}{c}(2) \\
\text { HBSC-SCL }\end{array}$ & $\begin{array}{c}(3) \\
\text { SDQ }\end{array}$ & $\begin{array}{c}(4) \\
\text { CES-DC }\end{array}$ & $\begin{array}{c}(5) \\
\text { SCARED }\end{array}$ \\
\hline \multicolumn{6}{|c|}{ Panel a: effect by age } \\
\hline $11 \mathrm{y} / \mathrm{o}$ & $\begin{array}{c}-0.213^{* * *} \\
(0.063)\end{array}$ & $\begin{array}{c}0.210^{* * *} \\
(0.067)\end{array}$ & $\begin{array}{c}0.240^{* * *} \\
(0.054)\end{array}$ & $\begin{array}{l}0.146^{* *} \\
(0.062)\end{array}$ & $\begin{array}{l}0.107^{*} \\
(0.064)\end{array}$ \\
\hline $12 \mathrm{y} / \mathrm{o}$ & $\begin{array}{c}-0.166^{* * *} \\
(0.052)\end{array}$ & $\begin{array}{c}0.141^{* * *} \\
(0.054)\end{array}$ & $\begin{array}{c}0.165^{* * *} \\
(0.046)\end{array}$ & $\begin{array}{l}0.109^{*} \\
(0.057)\end{array}$ & $\begin{array}{c}0.067 \\
(0.054)\end{array}$ \\
\hline $13 \mathrm{y} / \mathrm{o}$ & $\begin{array}{c}-0.150^{* * *} \\
(0.041)\end{array}$ & $\begin{array}{c}0.127^{* * *} \\
(0.042)\end{array}$ & $\begin{array}{c}0.128^{* * *} \\
(0.036)\end{array}$ & $\begin{array}{c}0.112^{* * *} \\
(0.042)\end{array}$ & $\begin{array}{c}0.056 \\
(0.041)\end{array}$ \\
\hline $14 \mathrm{y} / \mathrm{o}$ & $\begin{array}{c}-0.117^{* * *} \\
(0.034)\end{array}$ & $\begin{array}{c}0.090^{* *} \\
(0.035)\end{array}$ & $\begin{array}{c}0.109^{* * *} \\
(0.035)\end{array}$ & $\begin{array}{c}0.091^{* *} \\
(0.037)\end{array}$ & $\begin{array}{c}0.036 \\
(0.037)\end{array}$ \\
\hline $15 \mathrm{y} / \mathrm{o}$ & $\begin{array}{c}-0.088^{* * *} \\
(0.030)\end{array}$ & $\begin{array}{c}0.033 \\
(0.033)\end{array}$ & $\begin{array}{c}0.058 \\
(0.038)\end{array}$ & $\begin{array}{c}0.049 \\
(0.038)\end{array}$ & $\begin{array}{c}0.013 \\
(0.036)\end{array}$ \\
\hline $16 \mathrm{y} / \mathrm{o}$ & $\begin{array}{c}-0.066^{*} \\
(0.036)\end{array}$ & $\begin{array}{c}0.012 \\
(0.040)\end{array}$ & $\begin{array}{c}0.010 \\
(0.046)\end{array}$ & $\begin{array}{c}0.037 \\
(0.046)\end{array}$ & $\begin{array}{l}-0.008 \\
(0.044)\end{array}$ \\
\hline $17 \mathrm{y} / \mathrm{o}$ & $\begin{array}{c}-0.043 \\
(0.047)\end{array}$ & $\begin{array}{l}-0.018 \\
(0.045)\end{array}$ & $\begin{array}{c}-0.022 \\
(0.055)\end{array}$ & $\begin{array}{c}0.026 \\
(0.057)\end{array}$ & $\begin{array}{l}-0.016 \\
(0.057)\end{array}$ \\
\hline \multicolumn{6}{|c|}{ Panel b: effect by gender } \\
\hline boys & $\begin{array}{c}-0.161^{* * *} \\
(0.037)\end{array}$ & $\begin{array}{l}0.094^{* *} \\
(0.042)\end{array}$ & $\begin{array}{c}0.116^{* * *} \\
(0.041)\end{array}$ & $\begin{array}{l}0.104^{* *} \\
(0.044)\end{array}$ & $\begin{array}{l}0.078^{*} \\
(0.043)\end{array}$ \\
\hline girls & $\begin{array}{c}-0.065^{*} \\
(0.039)\end{array}$ & $\begin{array}{c}0.050 \\
(0.039)\end{array}$ & $\begin{array}{c}0.053 \\
(0.038)\end{array}$ & $\begin{array}{c}0.044 \\
(0.040)\end{array}$ & $\begin{array}{l}-0.016 \\
(0.043)\end{array}$ \\
\hline p-value & 0.024 & 0.323 & 0.069 & 0.196 & 0.060 \\
\hline \multicolumn{6}{|c|}{ Panel c: effect by living space per child } \\
\hline$<$ median & $\begin{array}{c}-0.182^{* * *} \\
(0.041)\end{array}$ & $\begin{array}{l}0.097^{* *} \\
(0.043)\end{array}$ & $\begin{array}{l}0.102^{* *} \\
(0.045)\end{array}$ & $\begin{array}{l}0.107^{* *} \\
(0.045)\end{array}$ & $\begin{array}{c}0.053 \\
(0.043)\end{array}$ \\
\hline$\geq$ median & $\begin{array}{c}-0.048 \\
(0.035)\end{array}$ & $\begin{array}{c}0.046 \\
(0.040)\end{array}$ & $\begin{array}{c}0.058 \\
(0.037)\end{array}$ & $\begin{array}{c}0.039 \\
(0.039)\end{array}$ & $\begin{array}{c}0.002 \\
(0.037)\end{array}$ \\
\hline p-value & 0.002 & 0.283 & 0.301 & 0.171 & 0.231 \\
\hline$N$ & 907 & 907 & 907 & 907 & 907 \\
\hline
\end{tabular}

Extended Data Table 3: Subgroup Analysis for the short-run impact of one additional week school closure on self-reported mental health Estimates for the effect of one additional week school closure on standardized measures (mean 0, std. 1) of adolescents' HRQoL and screening devices for mental health issues. Panel a) shows the results by age, Panel b) by gender, Panel c) by living space per child. The sample consists of $n=907$ 11-17year-old adolescents participating in the first COPSY wave (May 26 - June 10, 2020). 


\begin{tabular}{lccc}
\hline & $(1)$ & $(2)$ & $(3)$ \\
& Fear & Family & Friends \\
\hline Q2/2020 & -2.024 & $2.494^{* *}$ & $3.633^{*}$ \\
& $(2.559)$ & $(1.196)$ & $(2.165)$ \\
Q3/2020 & -1.981 & -0.492 & 1.949 \\
& $(2.026)$ & $(0.986)$ & $(1.568)$ \\
Q4/2020 & -1.065 & $2.233^{* *}$ & $3.448^{* *}$ \\
& $(2.184)$ & $(1.029)$ & $(1.587)$ \\
\hline$N$ & 9721 & 9721 & 9721 \\
\hline${ }^{*} p<0.1,{ }^{* *} p<0.05,{ }^{* * *} p<0.01$. &
\end{tabular}

Extended Data Table 4: Effect of one additional week of school closure on call volume Each column consists of one estimation estimating the effect of one additional week of school closure on call volume. One observation is the call volume of one age group, in one state, during one week. Call volume is the total duration of all calls mentioning fear, family problems, or problems with friends. Call volume is standardized for each topic such that it has a mean of 0 in 2019 and multiplied by 100 to interpret the coefficients as percentage points changes. Each regression has an indicator variable for the $2^{\text {nd }}, 3^{\text {rd }}$, and $4^{\text {th }}$ quarter which is interacted with our measure of predicted weeks of school closure that a age-state cohort has experienced so far. Further, each regression contains age group, state, and week fixed effects. In addition, each regression contains a dummy that is one for each week after March 16, 2020 and 0 for each week before. Robust standard errors are clustered on age group*state*quarter*year level and shown in parentheses. 